S. Kwok, M. Dopita, and R. Sutherland, eds.

\title{
The Sulphur Abundance in Planetary Nebulae
}

\author{
Mezak A. Ratag \\ Indonesian National Institute of Aeronautics 6 Space (LAPAN) \\ Jl. Junjunan 133, Bandung 40173, Indonesia
}

\section{Introduction}

Ratag et al (1997) determined the chemical abundances of $\mathrm{He}, \mathrm{O}, \mathrm{N}, \mathrm{Ne}, \mathrm{S}$, $\mathrm{Ar}$, and $\mathrm{Cl}$ in 110 planetary nebulae which are likely to be in the Galactic Bulge. The abundances are derived by employing theoretical nebular models as interpolation devices in establishing the ICFs. The overall agreement between the abundances obtained by employing the model-ICFs and those derived from the theoretical models is reasonably good. Based on this, it is of interest to study the reliability of various ICFs as well as to derive some alternative ICFs. The focus of the present study is sulphur.

\section{Data \& Method}

From the sample of planetary nebulae studied by Ratag et al. (1997), we selected those for which the [SII] doublet at about $6725 \AA$ and the [SIII] line at $\lambda 6312 \AA$ are detectable so that the corresponding ionic concentrations can be computed. The next criterion applied to the sample is the availibility of the $\mathrm{O}^{+}$ ionic as well as $O$ total abundances. In total, 42 planetary nebulae form the final sample. The sulphur abundances in those nebulae are then recomputed using the ionization correction factors (ICFs) previously proposed by Stasinska (1978) [see also Kingsburgh \& Barlow, 1994] and by Kwitter \& Henry (2001). The resulting abundances are denoted, respectively, by $(\mathrm{S} / \mathrm{H})_{S}$ and $(\mathrm{S} / \mathrm{H})_{K H}$.

$$
\begin{gathered}
(S / H)_{\mathrm{S}}=\left[1-\left[1-\left(O^{+} / O\right)^{3}\right]\right]^{-1 / 3}\left[S^{+}+S^{+2}\right] / H^{+} \\
(S / H)_{\mathrm{KH}}=\operatorname{dexp}\left(-0.017+0.18 \beta-0.11 \beta^{2}+0.072 \beta^{3}\right)\left[S^{+}+S^{+2}\right] / H^{+}
\end{gathered}
$$

where $\beta \equiv \log \left(\mathrm{O} / \mathrm{O}^{+}\right)$. The logarithmic abundances are then defined by $[\mathrm{S}]_{S}=$ $\log (\mathrm{S} / \mathrm{H})_{S}+12$ and $[\mathrm{S}]_{K H}=\log (\mathrm{S} / \mathrm{H})_{K H}+12$. For comparison, the sulphur abundances are also recalculated using the classical ICF proposed by Peimbert $\&$ Costero (1969). They are denoted by $[\mathrm{S}]_{\mathrm{PC}} \cdot[\mathrm{S}]_{\mathrm{PC}},[\mathrm{S}]_{\mathrm{S}}$ and $[\mathrm{S}]_{\mathrm{KH}}$ are plotted against the sulphur abundances [S] derived by Ratag et al (1997) in Figs. 1, 2 and 3. As shown in Fig. 1, the classical ICF proposed by Peimbert \& Costero (1969) clearly overestimates the total sulphur abundances. Figs. 2 and 3 indicate that both $[\mathrm{S}]_{S}$ and $[\mathrm{S}]_{K H}$ are, on average, lower than $[\mathrm{S}]$. 

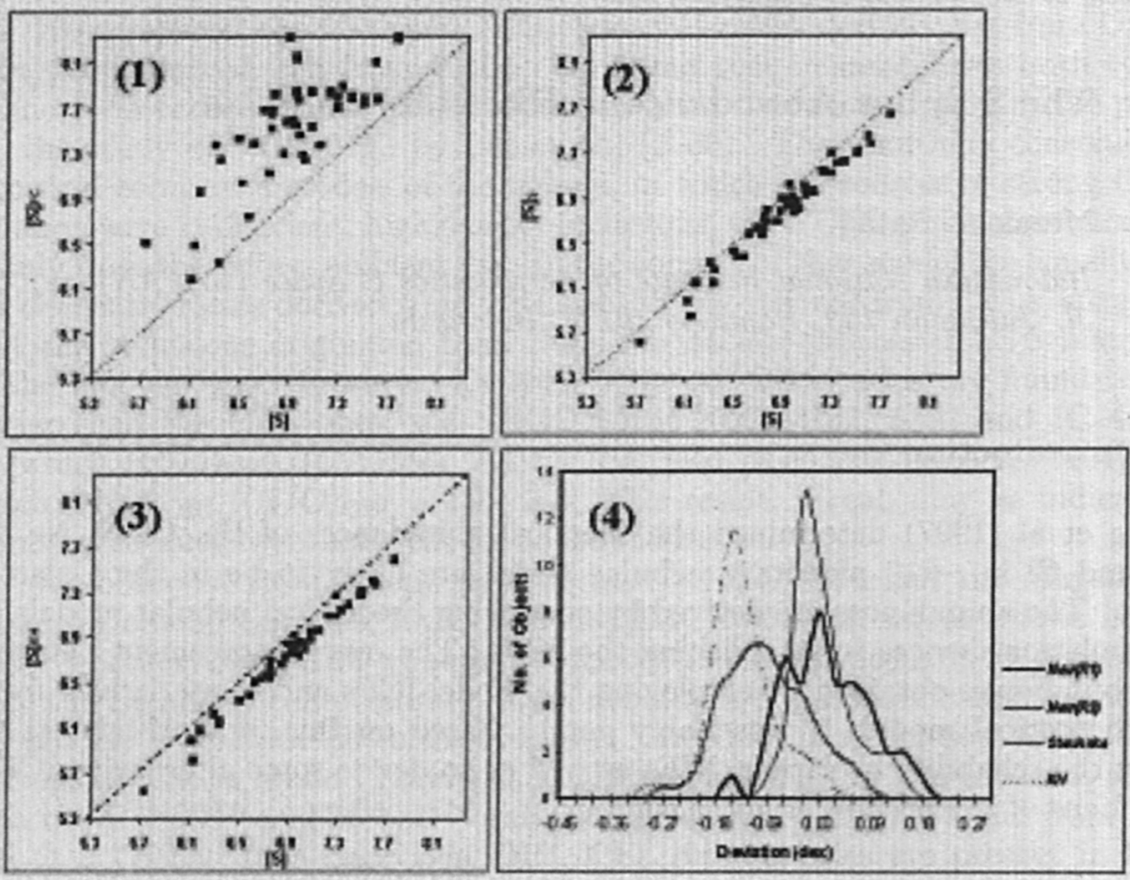

Figure 1. Comparison of sulphur abundances between different authors.

\section{Results}

Best fits to the $1: 1$ line can be obtained if $[\mathrm{S}]_{\mathrm{S}}$ and $[\mathrm{S}]_{\mathrm{KH}}$ are increased by, respectively, 0.1190 and $0.1639 \mathrm{dex}$. Thus, we have two new alternative formulae for sulphur abundance estimation based on equations (1) and (2), namely

$$
\begin{gathered}
(S / H)_{\mathrm{R} 1}=1.315\left[1-\left[1-\left(O^{+} / O\right)^{3}\right]\right]^{-1 / 3}\left[S^{+}+S^{+2}\right] / H^{+}, \text {and } \\
(S / H)_{\mathrm{R} 2}=1.458 \operatorname{dexp}\left(-0.017+0.18 \beta-0.11 \beta^{2}+0.072 \beta^{3}\right)\left[S^{+}+S^{+2}\right] / H^{+}
\end{gathered}
$$

Smoothed distributions of the differences between $[\mathrm{S}]_{\mathrm{R} 1}$ and $[\mathrm{S}]_{\mathrm{R} 2}$, and the sulphur abundances [S] determined by Ratag et al (1997) are shown in Fig. 4. The distributions for $[\mathrm{S}]_{\mathrm{S}}$ and $[\mathrm{S}]_{\mathrm{KH}}$ are displayed for comparisons. Figure 4 shows that both corrected equations (3) and (4) are comparable. The standard deviation ( of 0.076 dex for the R1-distribution and 0.057 dex for the R2-distribution, however, suggest that the R2 equation is slightly better.

\section{References}

Kingsburgh, R.L., \& Barlow, M.J. 1994, MNRAS, 271, 257

Kwitter, K.B., \& Henry, R.B.C. 2001, ApJ, 562, 804

Ratag, M.A., Pottasch, S.R., Dennefeld, M., Menzies, J. 1997, A\&AS, 126, 297

Stasinska, G. 1978, A\&A, 66, 257 